\title{
A Novel Treatment for Proximal Clavicle Fracture Combined With Sternoclavicular Dislocation
}

\section{Yadi Zhang ( $\nabla$ m13296813753@163.com )}

Cangzhou Hospital of integrated traditional Chinese medicine and Western medicine

\section{Baorui Xing}

Cangzhou Hospital of integrated traditional Chinese medicine and Western medicine

\section{Xiuxiu Hou}

Cangzhou Hospital of integrated traditional Chinese medicine and Western medicine Yunmei Li

Cangzhou Hospital of integrated traditional Chinese medicine and Western medicine

\section{Research Article}

Keywords: sternoclavicular joint, unstable, fracture, surgical repair

Posted Date: February 11th, 2022

DOI: https://doi.org/10.21203/rs.3.rs-1301029/v1

License: (c) (1) This work is licensed under a Creative Commons Attribution 4.0 International License. Read Full License 


\section{Abstract}

Purpose: The aim of this study was to evaluate the clinical results of sternoclavicular hook plate and anterior sternoclavicular ligament repair in proximal clavicle fractures.

Methods: Between October 2016 and December 2020, a total 16 patients ( 9 male and 7 female patient, with a mean age of $(42.25 \pm 10.24)$ years (range,25-56 years) with unstable proximal clavicle fractures (Throckmorton, type $\mathrm{D}$ ) were treated with sternoclavicular hook plate, and anterior sternoclavicular ligament repair. Affected side, cause of the injuries, dislocation type, associated injuries, interval between injury and surgery, and duration of follow-up were recorded. The outcomes were evaluated with radiographic assessment, American Shoulder and Elbow Surgeons' Form(ASES). All the patients were evaluated on postoperative 3rd, 6th, and 12th months.

Results: According to the ASES scoring system, the average score was $49.50 \pm 3.76$ (preoperative score),86.94 \pm 5.09 (3 months follow-up), 88.32 \pm 3.07 (6 months follow-up) and91.00 \pm 2.92 (12 months follow-up).Statistics differences was showed between preoperative and 3,6,12 months follow-up score of ASES SCJ score $(p<0.05)$. The postoperative physical function was better than the preoperative function.

Conclusion: Fixation using a sternoclavicular hook plate combined with repair of the ligament and capsule isrelatively easy to do; it provides micromotion, which is beneficial for the movement of the shoulder girdle. Our study indicates that open reduction and sternoclavicular hook plate fixation for the treatment of traumatic sternoclavicul-ar joint dislocations or fracture is a safe, relatively straightforward surgical procedure that can lead to satisfactory outcomes.

\section{Introduction}

The sternoclavicular joint (SCJ) is a freely moveable joint allowing for upward elevation, anteroposterior translation, and rotation along its long axis and contributes to scapulothoracic motion[1], It's the only synovial joint connecting the upper extremity and the trunk. The stability of the joint depends exclusively on the meniscus, joint capsule and the surrounding ligaments[2]. SCJ dislocation is a rare injury. The incidence of sternoclavicular dislocation is approximately $3 \%$ of the shoulder injuries.,while the incidence of proximal clavicle fractures have lower incidence than fracture of the middle third and distal clavicula fracture, accounting for $5-6 \%$ of the clavicle fractures $[3,4]$. However, physeal fratures and joint dislocation are not routinely divided into different injuries, which is a significant limitation of most research to date and therefore diluting the applicability of suggested treatment algorithms $[5,6]$. SCJ dislocation was presumably frequently missed, both clinically and radiographically.Kusnezov et al.report in their study group a rate of late diagnosis of more than 1 year in $71 \%$ of all cases[7]. With the widespread availability of CT scanning, it's now straight-forward to diagnose yet it is still frequently misseed.

Historically,a number of reconstruction techniques have been described.These all generally involve Kirschner wires[8], Steinmann pins[9], soft tissue reconstruction (suture,autologous tendon,allograft) [10, 11], plate techbiques[12], sternoclavicular temporary arthrodesis[13], sterno-clavicular buttress plate and 
sternoclavicular hook plate[14]. There is little literature describe on SCJ dislocation or fracture, but no gold standard has been establish.We describe our preferred surgical approach for the repair of unstable dislocation of the SCJ or fracture with sternoclavicular hook plate.Furthermore patient outcome and midterm follow-up results are reporeted.

\section{Methods}

\section{General Data}

All 16 cases of SCJ dislocation or fracture treated between October 2016 and December 2020 were included in the study. Informed written consent for publication of this report and accompanying figures was obtained from the patients. The hospital research ethics committee approved the study. Selection criteria included (1) dislocations that could not be reduced by nonoperative treatment and type $D$ fresh proximal clavicle fractures according to the Throckmorton classification (Fig 1.); (2) dislocations that were prone to recurrence during shoulder movement and cases with prominent skin lesions; and (3) patients without underlying diseases such as heart disease. Exclusion criteria. (1) Patients with brain injury or severe underlying chronic disease and, therefore, cannot tolerate the risk of surgery and anesthesia; (2) pathological fractures; (3) open fractures; (4) combined vascular or neurological injuries.

(5) The patient requested conservative treatment, even though closed reduction was pointless.all procedures were performed by the senior author after obtaining informed consent from all patients.

Conventional radiographs are not sensitive for posterior sternoclavicular dislocations, and computed tomography (CT) represents the imaging modality of choice[15, 16]. All patients underwent the standard preoperative assessment, including preoperative history, physical examination, plain radiograph of the clavicle, sternum and thorax completed with serendipity view and a CT scan of the thorax(Fig.2).

\section{Surgical Technique}

All patients were positioned supine with free draping of the ipsilateral arm on the operating table, and underwent general anesthesia.An hockey-stick shaped incision of approximately 8-10 cm length was made from the medial clavicle,via the sternoclavicular joint,and to the superior part of the sternal manubrium.Subcutaneous superficial fascia and deep fascia were incisionally incisional to expose the manureum sternum, sternoclavicular joint and proximal clavicular fracture, showing obvious fracture displacement.After the upper margin was exfoliated, the posterior sternal soft tissue was exfoliated along the posterior margin of the manubrium sternum with periosteum exfoliator.From above the sternal handle place(the diamond drill jig sternum has along the thread in the block at the back of the handle), in the centerline of the sternal handle drilling, drilling through the steel wire head end lock on the jig block slice, pull out of jig, steel wire, jig, the steel wire head end locked into the appropriate sternoclavicular hook plate, locked sternoclavicular hook plate through the sternocleidomastoid trailing edge.The sternum end of the sternum plate of the sternum was pierced through the hole through the traction of the wire wire(Fig.1). The proximal clavicle fracture was reduced. After satisfactory reduction, the plate was fixed on the clavicle with a common screw (for proximal clavicular fracture comminuted, it is recommended to 
use an Achilles tendon suture to strengthen the fixation), and a gasket and nut were installed on the hook thread of the patient.A large amount of normal saline w-as rinsed in the surgical area. After the instrument and auxiliary materials were checked correctly, a negative pressure drainage tube was placed in the surgical area, the skin was sutured layer by layer, and the incision was covered with sterile dressing.

\section{Postoperative management}

Three days after surgery, patients were allowed to do passive forward flexion and abduction of the shoulder joint under the guidance of the physical therapist. Depending on the degree of pain and postoperative X-ray and CT scan images, the range of motion could be gradually increased, and no weight-bearing exercise was allowed. Weight-bearing exercises were gradually started after 6 weeks postoperatively. Postoperative follow-up is every 4 weeks until the bone heals, and every 3 months after the bone heals, and exercise should be avoided for 12 weeks after surgery. The sternoclavicular plate can be removed at 12 months after surgery according to the clinical course (Fig 3 ).

\section{Outcome measures}

Postoperative function was evaluated using American Shoulder and Elbow Surgeons' Form(ASES) [17].The system is a 100-point system consisting of the patient's own assessment section (50\%) and the cumulative daily activities section (50\%).Patients were assessed for pain, stability, and daily activities.Part of the doctor's assessment is mobility, signs, strength tests, and stability. The higher the score, the better shoulder function.VAS was used to evaluate the pain scale[18]. The life function scale included 10 daily activities: dressing, combing hair, and going to the toilet.Placzek et al.[19] found that ASES score had low correlation with age and high credibility through studies.Patients were routinely followed at 2 weeks and 9-12 months postoperatively. No significant complications occurred during follow-up(Fig.4).

\section{Statistical analysis}

All statistical data were statistically analyzed using the statistical software SPSS 20.0 (Statistical Package for Social Sciences, SPSS Inc, Chicago, IL, USA) to calculate the results of each measure, and the values are expressed as mean \pm standard deviation. Count data were analyzed by t-test, and $\mathrm{P}<0.05$ was set as a statistically significant difference.

\section{Results}

There were 9 male patients and 7 female patients with a mean age of $(42.00 \pm 10.00)$ years.Four patients had unilateral dislocation, 12 patients had proximal clavicle fractures, and 1 of the 16 patients had old dislocation (more than 3 weeks). One patient had a rib fracture; mechanism of injury: 12 patients were in a car injury and 4 patients were injured by an impact object. Age, gender distribution, side involved, cause of injury, type of dislocation, associated injury, time between injury and surgery, and follow-up are shown

in Table 1. According to the ASES scoring system, the average score was $49.50 \pm 3.76$ (preoperative score), 
86.94 \pm 5.09 (3 months follow-up), 88.32 \pm 3.07 (6 months follow-up) and 91.00 \pm 2.92 (12 months followup).Statistics differences was showed between preoperative and 3,6,12 months follow-up score of ASES SCJ score $(p<0.05)$. The postoperative physical function was better than the preoperative function(Tab.2).

\section{Discussion}

The SCJ is a relatively unstable saddle synovial joint. Based on its anatomy, we can understand that the stability of the SCJ is maintained by its periarticular ligaments. Also the activity of the shoulder joint complex can cause the movement of the SCJ[20]. When the arm is raised $120^{\circ}$, the clavicular end of the sternoclavicular joint is posteriorly rotated $15^{\circ}$, raised $5^{\circ}$, and internally retracted $15^{\circ}$. The stability of the SCJ is maintained mainly by the posterior sternoclavicular ligament, whose rupture will lead to joint instability and posterior dislocation[21].

There is still no consensus regarding the treatment of sternoclavicular joint dislocation orfracture. Groh et al. report on a trial of closed reduction in 21 patients, which was effective in only eight patients[22].Previous studies have also shown that closed reduction of sternoclavicular dislocation has a high risk of redislocation[23].Even after successful closed reduction, residual sternoclavicular instability still exists, requiring subsequent secondary surgery[23].If closed reduction does not work,then open treatment should be considered.Various techniques have been proposed for the surgical management of the SCJ dislocation or fracture. Considering the structural characteristics of the SCJ, the application of kwire fixation will lead to the displacement of the k-wire due to the activity of the SCJ, which in turn will lead to the injury of the surrounding large vessels. The application of locking plate fixation of the SCJ will result in restricted movement of the joint and loss of most of its function. With increasing attention to the degree of postoperative pain and functional recovery, locking plate fixation of the SCJ has gradually been discouraged for this joint[24].The transfer of the sternocleidomastoid tendon or the subclavius tendon has been described[25]. The failure rate of tendon autografting may be related to the complexity of the technique, operation time and the site where the tendon is obtained [26, 27].

In this study, an sternoclavicular joint hook plate was used for the treatment of sternoclavicular joint injury. There are some advantages of the treatment using the sternoclavicular hook plate:1) The anterior medial side of the clavicle is fixed with cortical screws, which not only provides stability, but also allows the hook plate to provide a certain range of fretting when the sternoclavicular joint is active $₫ 2)$ Patients can exercise shoulder joint in the early stage after surgery $₫ 3$ ) Sternoclavicular dislocation with proximal clavicular fracture can be fixed to provide sufficient strength to maintain proximal clavicular fracture $\varangle 4$ ) The hook end of the plate passes behind the sternocleidomastoid muscle, crosses the upper margin of the manubrium sternum and penetrates from the manubrium sternum body, avoiding the risk of intraoperative damage to important blood vessels and lungs.

In our report, we performed incisional repositioning sternoclavicular hook plate fixation in 16 patients with SCJ dislocation and/or proximal clavicle fractures. Postoperatively, good shoulder motion was obtained 
with no discomfort at the 2-month postoperative follow-up.

There are some limitations in this study; SCJ dislocation and/or fracture is a rare lesion, the sample size of this study is small, and further expansion of the sample size in multicenter studies is needed to support our findings. Also, we did not have a randomized control group, and the specific advantages and disadvantages of this procedure over other procedures need to be further investigated.

\section{Conclusion}

Fixation with a sternoclavicular hook plate is relatively easy, and it provides micromovement that facilitates shoulder girdle movement. Our study shows that adopting open reduction and sternoclavicular hook plate fixation for traumatic sternoclavicular dislocation or fracture is a safe and relatively simple procedure that can achieve satisfactory results.

\section{Declarations}

Thanks for the contribution of the tracking system.

\section{Acknowledgements}

We would like to thank Drs Xiaoming Li for insightful conversations.

\section{Authors' contributions}

Yadi Zhang designed this study, prepared the manuscript, tables and figures and have read and approved the final manuscript.,Baorui Xing, Xiuxiu Hou, Yunmei Li collected and/or rated the data, read and approved the final manuscript.

\section{Funding}

None

\section{Availability of data and materials}

The patients' dataset are confidential and are privately held for patients confidentiality safeguard. As such, the datasets generated and/or analysed during the current study are not publicly available but are available from the corresponding author on reasonable request.

\section{Ethics approval and consent to participate}

This study was approved by the medical ethics review board of Cangzhou Hospital of integrated traditional Chinese medicine and Western medicine.

All methods in the study were carried out in accordance with the Helsinki guidelines and declaration. 
All procedures were undertaken by the senior author after obtaining informed consent for all patients.

\section{Consent for publication}

We acquired informed written consent for publication of this report and accompanying images from the patients.

\section{Competing interests}

No competing interest to report.

\section{Reference}

1. Armstrong, L.J.O. Alison, and Trauma,(2016) Disorders of the sternoclavicular joint. J Orthopaedics \& Trauma,32(3):p324-335.

2. Lee, J.T., et al., (2014) Posterior Sternoclavicular Joint Injuries in Skeletally Immature Patients. J Pediatr Orthop, 34(4): p. 369-375.

3. Sidhu, V.S., et al., (2015) The operative outcomes of displaced medial-end clavicle fractures.J Shoulder\&Elbow Surgery, 24(11): p. 1728-1734.

4. Goldfarb, C.A., et al., (2001) Retrosternal displacement after physeal fracture of the medial clavicle in children.J Bone\&Joint Surgery Britsh Volume, 83(8): p. 1168-72.

5. Bicos, J. and G.P.J.C.i.S.M. Nicholson,(2003) Treatment and results of sternoclavicular joint injuries. $\mathrm{J}$ Clinics in Sports Medicine,22(2): p. 359-370.

6. Rajaratnam, S., M. Kerins, and L.J.C.A. Apthorp, (2002) Posterior dislocation of the sternoclavicular joint: A case report and review of the clinical anatomy of the region. J Clinical Anatomy,15(2): p. 10811.

7. Kusnezov, N., et al., (2015) Sternoclavicular Reconstruction in the Young Active Patient: Risk Factor Analysis and Clinical Outcomes at Short-Term Follow-Up. J Orthop Trauma,30(4): p. e111.

8. Wirth, M.A. and C.A.J.J.o.t.A.A.o.O.S. Rockwood, (1996) Acute and Chronic Traumatic Injuries of the Sternoclavicular Joint. J Am Acad Orthop,4(5): p. 268.

9. Groh, G.I. and M.A.J.J.o.t.A.A.o.O.S. Wirth, (2011) Management of traumatic sternoclavicular joint injuries. J Am Acad Orthop,19(1): p. 1-7.

10. Goost, H., et al., (2015) Surgical treatment of sternoclavicular joint instability with tenodesis.J Opera Orthop Und Traumatologie, 27(4):p.134-143.

11. Kohei,Kawaguchi,Sayo,Tanaka,(2015) Outcomes of augmented allograft figure-of-eight sternoclavicular joint reconstruction. J Shoulder \& Elbow Surgery,24(6): p. 902-907.

12. Shuler, F.D. and N.J.O. Pappas, (2008)Treatment of posterior sternoclavicular dislocation with locking plate osteosynthesis. J Orthop,31(3): p. 273. 
13. Quispe, J.C., et al.,(2016) Transarticular plating for acute posterior sternoclavicular joint dislocations: a valid treatment option? J International Orthopaedics,40(7): p. 1503-1508.

14. Chuanyi, et al.,(2017) Efficacy analysis of a novel sternoclavicular hook plate for treatment of unstable sternoclavicular joint dislocation or fracture. J Orthop Surgery,25(1):p.1-7.

15. Hoekzema, N., et al.,(2008) Posterior sternoclavicular joint dislocation.J Canasian Surgery, 51(1): p. 19-20.

16. Doss, A., et al.,(2005) Posterior sternoclavicular joint dislocation in children-role of spiral computed tomography. J Pediatric Emergency,21(5): p. 325.

17. Michener, A,Lor et al.(2002) American Shoulder and Elbow Surgrons StandardizedShoulder Assessment Form,patent self-report section:Reliability,validity, and responsiveness.Journal of Shoulder \& Eblow Surgrey.J Shoulder\&Elbow Surgery,11(6):587-594.

18. Ahmad, Alghadir, Shahnawaz et al. The Development and Validation of Hundred Paisa Pain Scale for Measuring Musculoskeletal Pain:A Prospective Observational Study. Medicine.2015.DOI:10.1097/MD.0000000000001162.

19. Placzek, R.Lindner, M. S.Deuretzbacher, G.(2004) Therapy for chronic radial epicondylitis withbotulinum toxin A - A therapy trial with a 2-year-follow-up.DOI:10.1055/s-2004-832407

20. Imam, M.A., et al.,(2018) The unicortical sternoclavicular joint reconstruction using synthetic graft. J Shoulder\&Eblow,DOI:10.1177/1758573218790964.

21. Spencer, E.E., et al., Hughes RE. (2002) Ligamentous restraints to anterior and posterior translation of the sternoclavicular joint. J Shoulder\&Elbow Surgery,11(1): p. 43-47.

22. Groh, G.I., et al., (2011) Treatment of traumatic posterior sternoclavicular dislocations. J Shoulder\&Elbow Surgery, 20(1): p. 107-113.

23. Laffosse, J.M., et al.,(2010) Posterior dislocation of the sternoclavicular joint and epiphyseal disruption of the medial clavicle with posterior displacement in sports participants.J Bone\&Joint Surgery British, 92(1): p. 103-9.

24. Laurencin, C.T., et al.,(1993) Dislocation of the sternoclavicular joint. Evaluation using paraxial computed tomographic reconstruction.J Orhop Rev, 22(1): p. 101-103.

25. Eskola, A., et al.,(1989) Operation for old sternoclavicular dislocation. Results in 12 cases.J Bone\&Joint Surgery British, 71(1): p. 63-5.

26. Martetschlger, F., et al.,(2015) Novel technique for sternoclavicular joint reconstruction using a gracilis tendon autograft.J Knee Surg Sports Traumatol A rthrosc, 24(7): p. 2225.

27. Barth, E. and R.J.A.O.S. Hagen, (2009) Surgical Treatment of Dislocations of the Sternoclavicular Joint.J Acta Orthopaedica Scandinavica,54(5):p.746-747.

\section{Tables}

Table 1 Patient characteristics 


\begin{tabular}{|c|c|c|c|c|c|c|}
\hline Patient & Gender & Age(years) & Side & Injury Mechanism & Time to Surgery & $\begin{array}{l}\text { Follow-up } \\
\text { (months) }\end{array}$ \\
\hline 1 & $\mathrm{~F}$ & 49 & $\mathrm{~L}$ & Road traffic accident & 1 week & 14 \\
\hline 2 & $M$ & 37 & L & Bike accident & 2 days & 12 \\
\hline 3 & M & 52 & $\mathrm{R}$ & Bike accident & 1 week & 18 \\
\hline 4 & $\mathrm{~F}$ & 48 & $\mathrm{~L}$ & Crashing object & 3days & 16 \\
\hline 5 & $\mathrm{~F}$ & 41 & $\mathrm{R}$ & Road traffic accident & 5 days & 21 \\
\hline 6 & M & 25 & $\mathrm{R}$ & Road traffic accident & 4 weeks & 23 \\
\hline 7 & M & 56 & L & Crashing object & 5 days & 13 \\
\hline 8 & $\mathrm{~F}$ & 34 & $\mathrm{R}$ & Crashing object & 3days & 13 \\
\hline 9 & $\mathrm{~F}$ & 42 & L & Road traffic accident & 6 days & 12 \\
\hline 10 & M & 44 & $\mathrm{R}$ & Bike accident & 3days & 14 \\
\hline 11 & $\mathrm{~F}$ & 28 & $\mathrm{R}$ & Road traffic accident & 1 week & 13 \\
\hline 12 & M & 27 & L & Bike accident & 3days & 15 \\
\hline 13 & M & 56 & $\mathrm{R}$ & Crashing object & 5 days & 18 \\
\hline 14 & M & 53 & L & Road traffic accident & 1 week & 21 \\
\hline 15 & $\mathrm{~F}$ & 48 & $\mathrm{R}$ & Bike accident & 1day & 13 \\
\hline 16 & M & 36 & $\mathrm{~L}$ & Bike accident & 5 days & 13 \\
\hline
\end{tabular}

Table 2 American Shoulder and Elbow Surgeons' Form(ASES) sternoclavicular joint of preoperation, $3,6,12$ months follow-up. 


\begin{tabular}{|c|c|c|c|c|}
\hline Patient & $\begin{array}{l}\text { ASES Score } \\
\text { (pre-op) }\end{array}$ & $\begin{array}{l}\text { ASES Score } \\
\text { (3 months post-op) }\end{array}$ & $\begin{array}{l}\text { ASES Score } \\
\text { (6 months post-op) }\end{array}$ & $\begin{array}{l}\text { ASES Score } \\
\text { (12 months post-op) }\end{array}$ \\
\hline 1 & 46 & 80 & 86 & 93 \\
\hline 2 & 53 & 86 & 90 & 90 \\
\hline 3 & 48 & 93 & 93 & 93 \\
\hline 4 & 60 & 83 & 85 & 85 \\
\hline 5 & 45 & 92 & 93 & 93 \\
\hline 6 & 46 & 80 & 85 & 90 \\
\hline 7 & 50 & 90 & 90 & 90 \\
\hline 8 & 52 & 85 & 86 & 92 \\
\hline 9 & 47 & 85 & 92 & 90 \\
\hline 10 & 52 & 93 & 90 & 95 \\
\hline 11 & 49 & 84 & 86 & 95 \\
\hline 12 & 48 & 92 & 91 & 92 \\
\hline 13 & 48 & 93 & 86 & 85 \\
\hline 14 & 46 & 83 & 85 & 93 \\
\hline 15 & 50 & 92 & 85 & 90 \\
\hline 16 & 52 & 80 & 90 & 90 \\
\hline Mean $\pm s$ & $49.50 \pm 3.76$ & $86.94 \pm 5.09$ & $88.32 \pm 3.07$ & $91.00 \pm 2.92$ \\
\hline $\mathrm{t}$ & & 23.659 & 31.983 & 34.867 \\
\hline $\mathrm{p}$ & & $0.000^{1}$ & $0.000^{2}$ & $0.000^{3}$ \\
\hline
\end{tabular}

Figures 


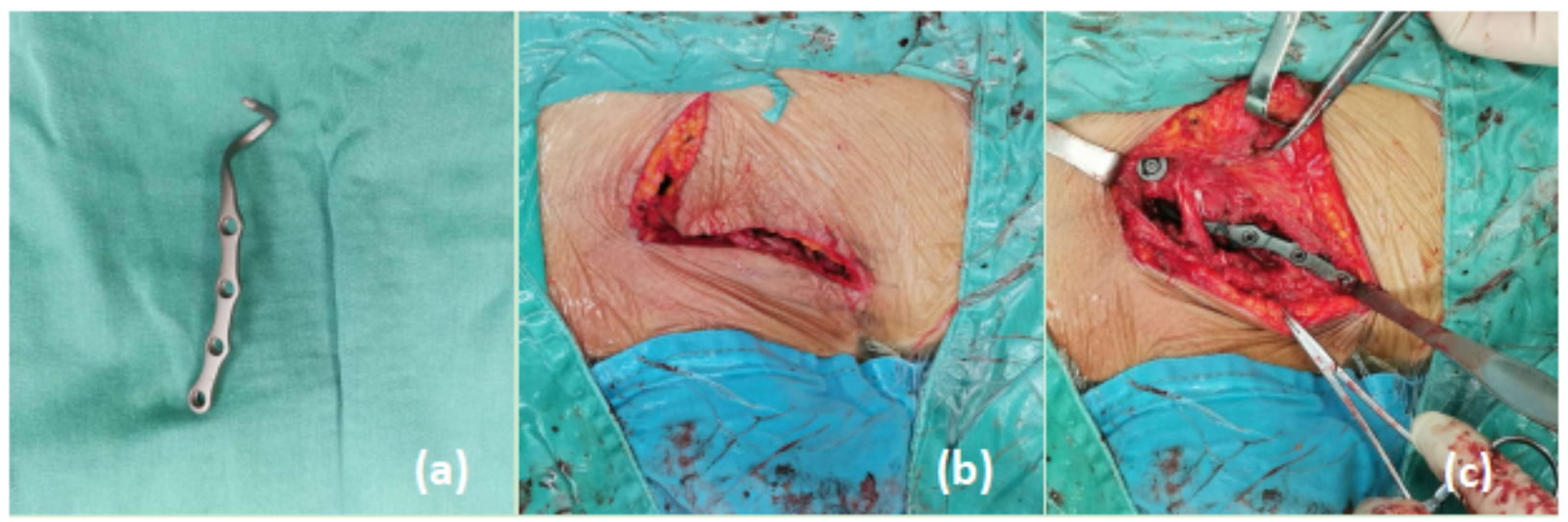

\section{Figure 1}

(a) Morphology of the plate, (b) anterior dislocation of the right sternoclavicular joint with an hockey-stick shaped incision, (c) from the rear of sternocleidomastoid, the hook end of sternocleidomastoid plate was fixed through the sternum stalk, and the proximal clavicular cortical bone screw was fixed.

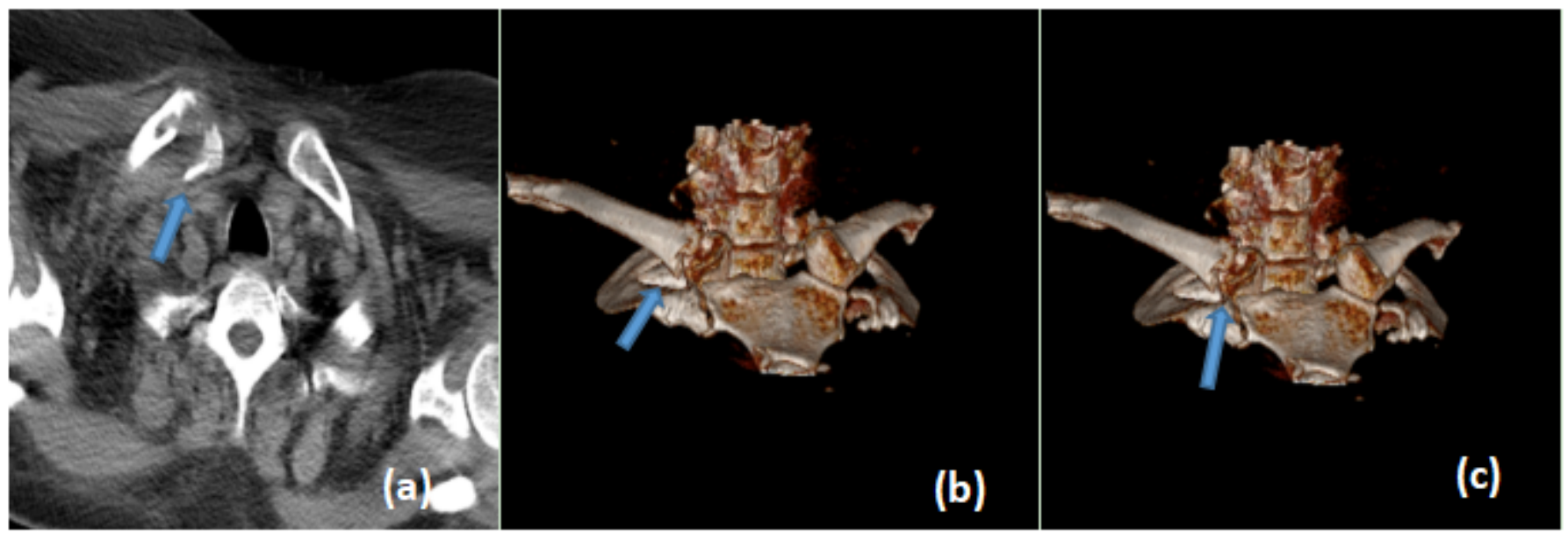

\section{Figure 2}

(a) Preoperative CT scan image show a right anterior dislocation of the sternoclavicular joint and proximal clavicle fracture, (b) Preoperative three-dimensional CT scan image, (c) Proximal end of the clavicle was displaced anteriorly and proximal clavicle fracture. 


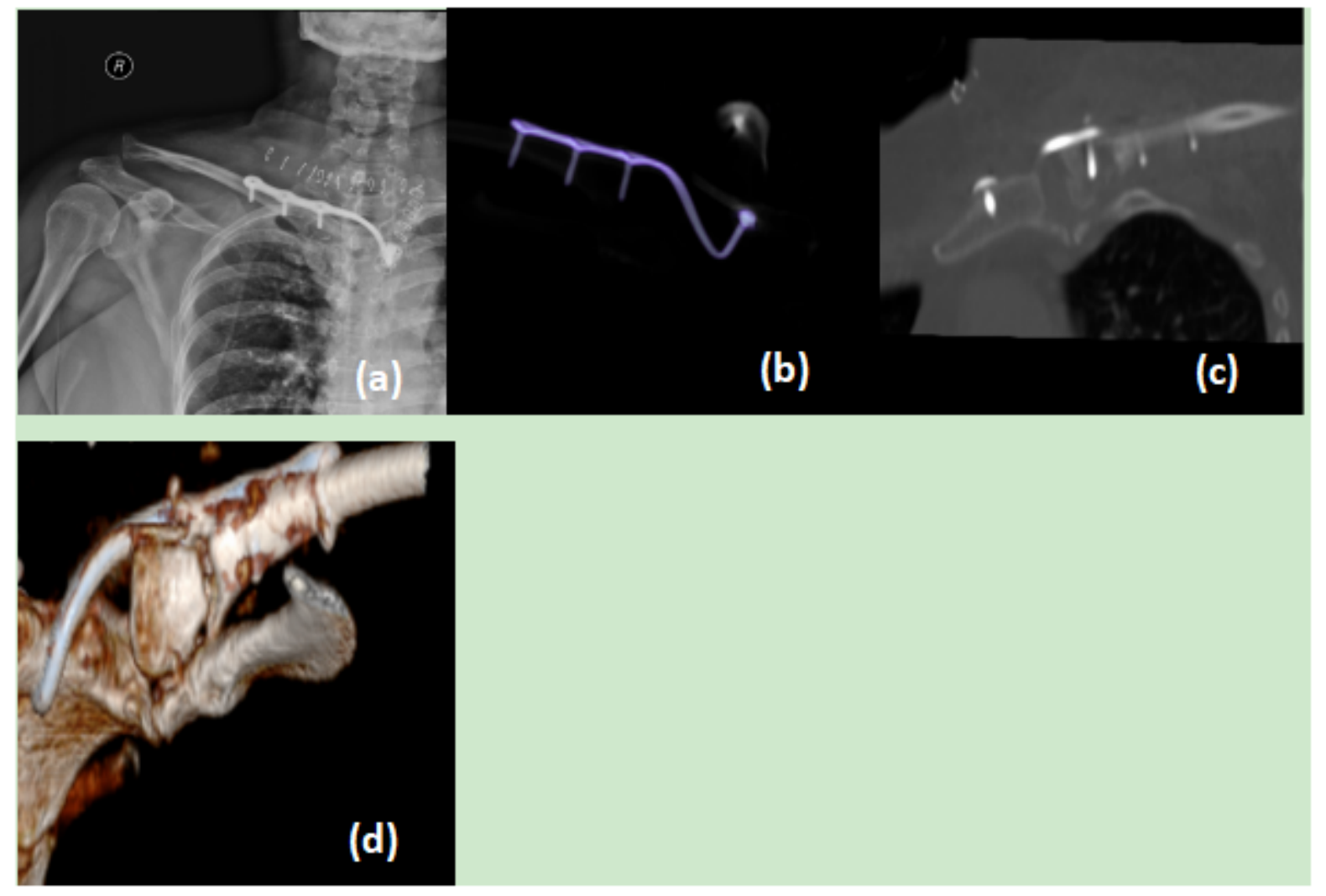

\section{Figure 3}

(a),(b)The postoperative X-ray image showed the well reduction by CanHSH sternoclavicular hook plate, (c),(d) postoperativeCT scan and three-dimensional CT scan of the patient after 3 months follow up. 


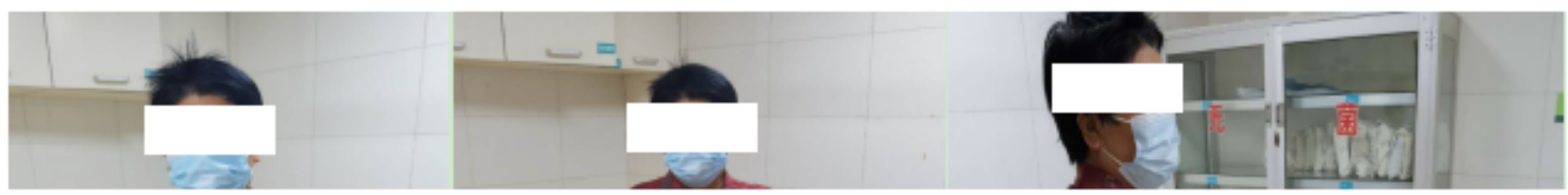

\section{Figure 4}

Functional outcome 3 months after reconstruction of anterior sternoclavicular joint Dislocation and proximal clavicle fracture. 